\title{
THE THERMOLUMINESCENCE OF LUNAR SAMPLES
}

\author{
G. F. J. GARLICK and IRENE ROBINSON \\ Department of Physics, University of Hull, England
}

\begin{abstract}
Studies of thermoluminescence emission from lunar samples show that at lunar day surface temperatures and even at $18^{\circ} \mathrm{C}$ the trapped electron responsible are decaying to their ground states by a non-thermal process. Data from various workers show similar characteristics with respect to this process. They show that thermoluminescence cannot be used as a technique for dating lunar samples (or terrestrial plagioclases) over any significant geological period. A quantitative model for the non-thermal process is proposed.
\end{abstract}

\section{Introduction}

Minerals in which there are luminescent components lend themselves to the technique of dating by means of thermoluminescence experiments. The electrons originally excited into metastable states or traps in the material by radioactive emissions or by other source such as cosmic rays may be released and return to normal ground states with emission of visible or near visible radiation if a sample is heated. If heating is at a uniform rate (or at some other controlled rate) then the so called 'thermoluminescence' curves can be quantitatively interpreted.

Since electron traps can themselves be due to defects in solids induced by high energy particles (radiation damage) the distribution of thermal activation energies (or trap depths) and the thermal stability of the traps themselves can be explored as a tool for analysis of the past radiation history of the sample. These aspects of thermoluminescence in minerals were thoroughly discussed at the Spoleto conference in 1968 [1]. In this paper we are concerned with the application of thermoluminescence studies to lunar material recovered in the Apollo 11 and 12 missions.

It was found by several different groups $[2,3,4,5,6]$ that the light sum of thermoluminescence obtained by warming Apollo 11 samples in the dark, and with no laboratory excitation, was many orders of magnitude below that expected from the accumulated dose of excitation from radioactive and cosmic ray sources on the lunar surface. Luminescence in lunar materials is of low efficiency $\left(\approx 10^{-5} \mathrm{~W}\right.$ output per watt input) but even this cannot account for the low thermoluminescence yields. It therefore became evident that trapped electrons in lunar samples were continuously 'leaking away' from the trapping states. Some authors attributed this to the thermal activation processes normally responsible for escape of trapped electrons which follow a Boltzmann type probability relation:

$$
p=s \exp (-E / k T)
$$

$s$ being the probability of escape per second, a constant $\left(\approx 10^{8}-10^{9}\right.$ for many silicates) $E$ the thermal activation energy (often termed the trap depth), $k$ being Boltzmann's constant and $T$ the absolute temperature. Thus electrons in deep traps ( $E$ large) will 
be much more stable than those in shallow traps ( $E$ small). While the high day temperature of the lunar surface $\left(\approx 120^{\circ} \mathrm{C}\right)$ could be invoked as a strong factor in the leakage process we were impressed by the fact that the rate of leakage was similar at terrestrial ambient temperatures around $20^{\circ} \mathrm{C}$ even in the case of deep traps. We therefore attempted to analyse the various data from our own measurements and those of other workers. This work and a theoretical analysis of the data form the substance of the following sections.

\section{Experimental Investigations}

\section{A. EXPERIMENTAL PROCEDURES}

In our thermoluminescence experiments samples of lunar material and of terrestrial plagioclases were used since the thermoluminescence characteristics and trapped electron leakage effects were found to be similar for the latter to those of lunar samples. Each selected specimen was mounted as a thin layer of dust in a light tight vacuum system on a 'finger' the temperature of which could be raised from room temperature

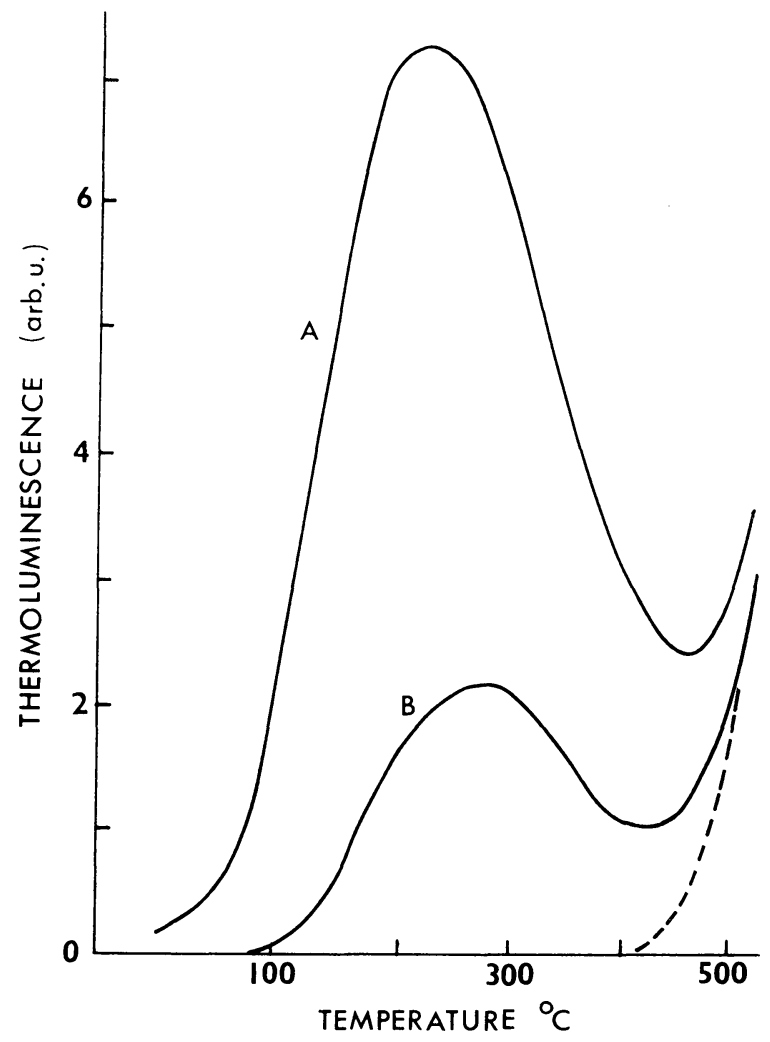

Fig. 1. Thermoluminescence curves of the plagioclase labradorite showing leakage of trapped electrons. A-Curve obtained after $16 \mathrm{~min}$ in dark at $18^{\circ} \mathrm{C}$ after $10^{7} \mathrm{rad}$ of $30 \mathrm{kV}$ X-irradiation. B-Curve obtained after $45 \mathrm{~h}$ in dark at $18^{\circ} \mathrm{C}$ after $10^{-} \mathrm{rad}$ of $30 \mathrm{kV}$ X-irradiation. 
at a uniform rate of $1 \mathrm{~K}$ per $\mathrm{s}$. The samples were viewed by a selected photomultiplier with visible or near infrared response. As an excitation source a $30 \mathrm{keV} \mathrm{X-ray} \mathrm{beam}$ was used and all samples received the same dose of $10^{7} \mathrm{rad}$. After excitation samples were held in the dark for a specified time and then warmed to obtain from the thermoluminescence curves the number of trapped electrons still remaining in the traps of different depths. These are the kinds of experiment arrangement and procedure adopted by the other workers whose data we incorporated in our analysis.

\section{B. EXPERIMENTAL RESULTS}

Since there are plenty of examples of the kinds of thermoluminescence curves obtained for Apollo 11 and 12 samples after laboratory excitation by X-rays or $\beta$-rays $[2,3,4]$ reference may be made to the respective publications. We give, however, an example of decay of trapped electrons after excitation of a terrestrial plagioclase, labradorite, by presenting in Figure 1 the thermoluminescence curves obtained by waiting for $16 \mathrm{~min}$ and for $45 \mathrm{~h}$ in the dark at $18^{\circ} \mathrm{C}$ respectively after the same initial excitation of $10^{7} \mathrm{rad}$. of $30 \mathrm{keV} \mathrm{X}$-rays. The loss of trapped electrons is very evident. Even the higher temperature regions of the curve (i.e. deep traps) are reduced.

In order to present in a concise way the various data from our experiments and those of other workers we have measured the height of a thermoluminescence curve at selected positions on the temperature abscissa as a function of the time the sample was held in the dark (at a selected lower temperature) before warming it to produce the curve. Figure 2 gives the curve height vs. time graphs so obtained. It is very notice-

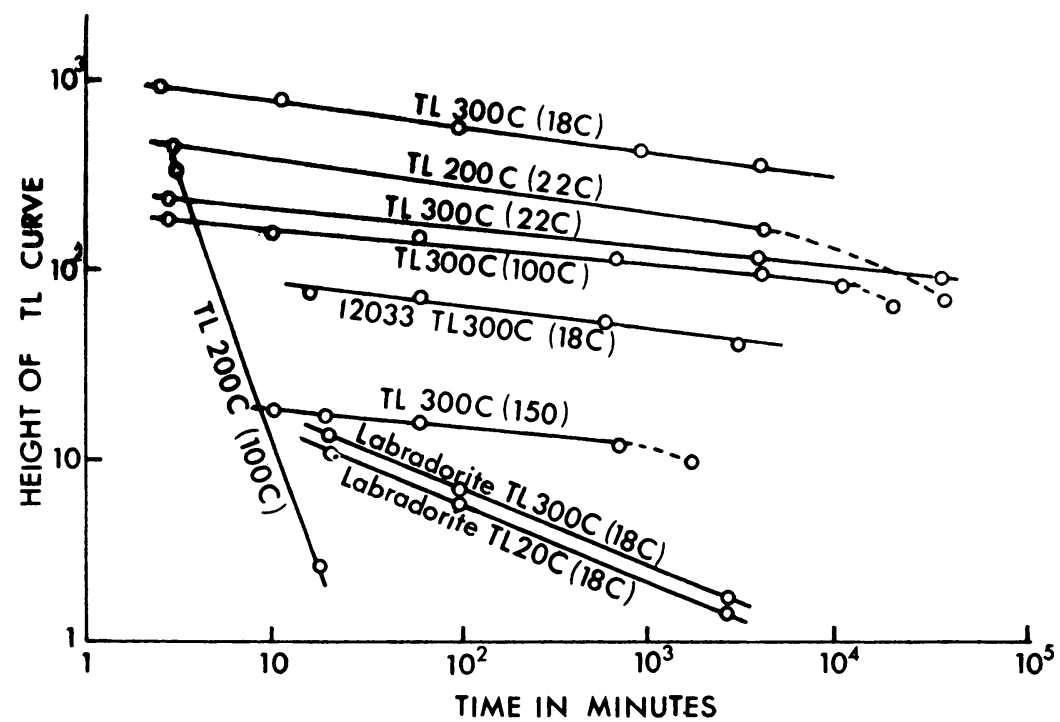

Fig. 2. Decay of thermoluminescence curves due to retention of samples at selected fixed temperatures for different times before warming. Point on thermoluminescence curve shown by first temperature on each curve: retention temperature shown in brackets. Curves for decay at $22^{\circ}$ and $100^{\circ} \mathrm{C}$ are from Dalrymple and Doell[ $\left.{ }^{3}\right]$ : curve for decay at $150^{\circ} \mathrm{C}$ is from Hoyt et al. $\left[^{2}\right]$ : both data are for Apollo 11 samples. 
able that in all cases but one the slopes are very similar for the lunar samples and independent of the thermoluminescence temperature chosen. In the one exceptional case of the decay for the $200^{\circ} \mathrm{C}$ curve region when the sample is held at $100^{\circ} \mathrm{C}$ the slope is large and decay rapid. We conclude that this is the consequence of a predominant thermal activation process.

The decay curves of Figure 2 for the terrestrial labradorite show similar behaviour but the larger slopes indicate a more rapid leakage of trapped electrons.

\section{Discussion of Results}

From the data of Figure 2 we conclude that there are two processes responsible for the loss of trapped electrons in lunar samples and in terrestrial plagioclases, one the thermal activation process (represented by Equation (1)) and the other a non-thermal leakage which affects electrons in traps over a wide range of depths or activation energies. The occurrence of the latter process means that we cannot use the thermoluminescence experiments as a dating technique in any straightforward way since only very small residual amounts of trapped electrons are found by the time the sample is available after retrieval from the lunar surface ( $\approx 9$ weeks).

In order to provide theoretical data on the residues of trapped electrons to be expected when only thermal activation processes are present and when distributions of trap depths are very large we have made a theoretical analysis for a uniform distribution of trap depths. If we use Equation (1) and assume first order kinetics the fraction $n / n_{0}$ of trapped electrons remaining at a given time after excitation ceases, and at a given sample temperature is given by

$$
n / n_{0}=\exp \{-s \exp (-E / k T) \cdot t\}
$$

Families of curves for $n_{0}$ constant with $E$ are given in Figure 3 assuming the constant $s=10^{9} \mathrm{~s}^{-1}$. It is a simple matter to shift such curves to accommodate other $s$ values.

The striking feature of such a relation is that for a given temperature and decay time all electrons in traps shallower than a given depth have escaped the other trapping states of greater depth remaining saturated. This means that for thermal activation alone the thermoluminescence curve decay shown in Figure 1 should be markedly different at different points along the temperature abscissa whereas Figure 2 shows for various samples little variation in the rates of decay with the temperature unless the thermoluminescence temperature is near to the temperature at which decay takes place. This supports our conclusion that a non thermal leakage process is present. The next task is to explain why a linear decay (i.e. power law) is displayed by he straight line graphs of Figure 2.

Non-thermal leakage of trapped electrons is most easily explained if a model as shown in Figure 4 is adopted. Any overlap of wave functions of trapped electrons and those applicable to the ground state energy level will give a finite transition probability. If each trap is simply associated with a neighbouring ground state then the 


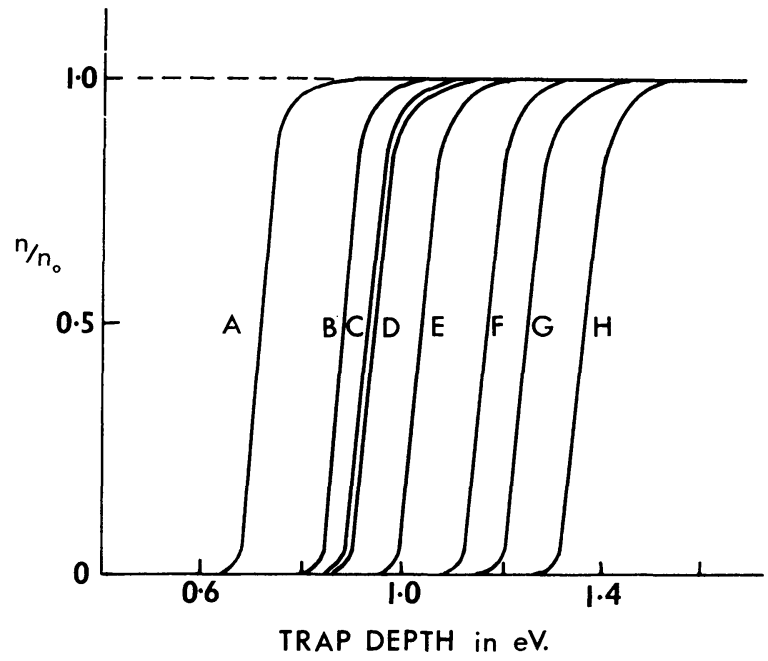

Fig. 3. Change in distribution of trapped electrons with time for different sample temperatures according to Equation (1) with $s=10^{9} \mathrm{~s}^{-1} \mathrm{~A}$-After $10 \mathrm{~min}$ at $300 \mathrm{~K}$ (same curve is 10 weeks at $300 \mathrm{~K}$ for $s=10^{5} \mathrm{~s}^{-1}$ ); B-After 1 week at $300 \mathrm{~K}$; C-After 10 weeks at $300 \mathrm{~K}$; D-After $10 \mathrm{~min}$ at $400 \mathrm{~K}$; E-After $10 \mathrm{yr}$ at $300 \mathrm{~K} ; \mathrm{F}$-After 1 week at $400 \mathrm{~K}$; G-After 10 weeks at $400 \mathrm{~K} ; \mathrm{H}$-After $10 \mathrm{yr}$ at $400 \mathrm{~K}$.
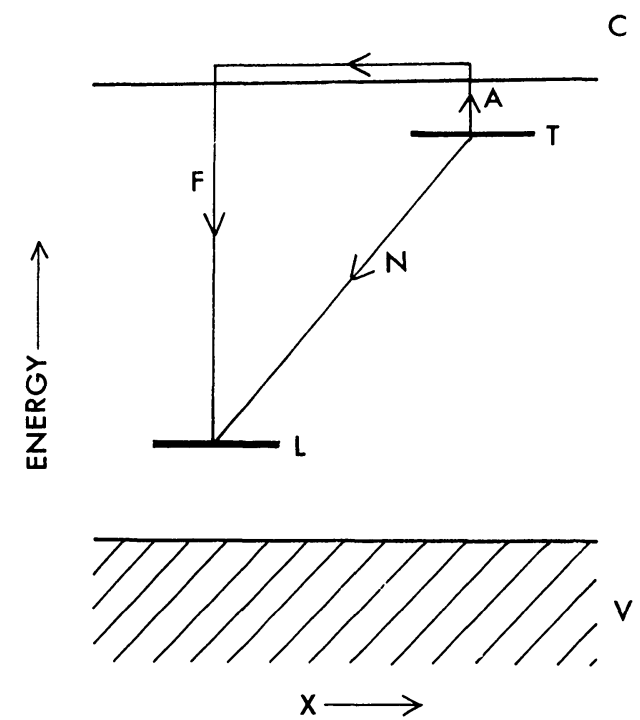

Fig. 4. Energy band model for non-thermal loss of trapped electrons in plagioclase. A-Thermal activation process; $\mathrm{C}$-Conduction band of host crystal lattice; F-Fluorescence transition in luminescence centre; L-Luminescence centre; $\mathrm{N}$-Non-thermal recombination of trapped electron with luminescence centre; $\mathrm{T}$-Electron trap; V-Valence band of host crystal lattice; $\mathrm{X}$-Spatial coordinate in host crystal lattice. 
decay of trapped electrons will be given by first order kinetics

$$
\mathrm{d} n / d \mathrm{t}=-\alpha n
$$

where $\alpha$ is the decay constant determined by the matrix element for the transition. However, if there is in the sample a random spatial distribution of trapping states and ground states the decay will be a complicated sum of exponentials. A similar situation has already been quantitatively treated by Thomas et al. [7] in explaining the luminescence emission spectra of GaP and other solids, known as the 'distant pair' theory. We shall not reiterate that analysis here but simply state that the resulting decay will follow a simple power law relation.

Turning to another facet of thermoluminescence studies on lunar samples it has been found both for Apollo 11 and Apollo 12 core samples that the residual thermoluminescence increases with the depth of the core sample below the lunar surface $[2,8]$. This might imply that thermal activation is playing a dominant part in the decay of trapped electrons in surface layers. However, the albedo of such core samples (measured in the laboratory) also shows an increase with depth of core sample [9]. This would give an apparent increased thermoluminescence since optical absorption is decreasing with the depth from which the sample was taken. Since thermoluminescence also depends on the intrinsic luminescence efficiency of a sample the latter may also be higher for depths of more than a $\mathrm{cm}$ or so due to the smaller damage in the sample from cosmic radiation. It is clear that interpretation of thermoluminescence for core samples must necessitate corrections for differences in sample opacity and luminescence efficiency.

\section{References}

[1] McDougall, D. J. (ed.): 1968, Thermoluminescence of Geological Materials, Academic Press.

[2] Hoyt, H. P., Kardos, J. L., Miyajima, M., Seitz, M. G., Sun, S. S., Walker, R. M., and Wittels, M. C.: 1970, Proc. Apollo 11 Lunar Sci. Conf. Geochim. Cosmochim. Acta Suppl. 1 3, 2269.

[3] Dalrymple, G. B. and Doell, R. R.: 1970, Proc. Apollo 11 Lunar Sci. Conf. Geochim. Cosmochim. Acta Suppl. 1 3, 2081.

[4] Geake, J. E., Dollfus, A., Garlick, G. F. J., Lamb, W., Walker, G., Steigmann, G. A., and Titulaer, C.: 1970, Proc. Apollo 11 Lunar Sci. Conf. Geochim. Cosmochim. Acta Suppl. 1 3, 2127.

[5] Nash, D. B. and Greer, R. T.: 1970, Proc. Apollo 11 Lunar Sci. Conf. Geochim. Cosmochim. Acta. Suppl. 1 3, 2341.

[6] Blair, J. M. and Edgington, J. A.: 1970, Proc. Apollo 11 Lunar Sci. Conf. Geochim. Cosmochim. Acta. Suppl. 1 3, 2001.

[7] Thomas, D. G., Hopfield, J. J., and Colbow K.: 1964, Radiative Recombination in Semiconductors 67.

[8] Hoyt, H., Kardos, J., Miyajima, M., Walker, R., and Zimmerman, D.: Proc. Apollo 12 Lunar Sci. Conf., MIT Press (in press).

[9] Nash, D. B. (unpublished work). 